\title{
The Combinatorialization of Linear Recurrences
}

\author{
Arthur T. Benjamin \\ Harvey Mudd College \\ Claremont, CA, USA \\ benjamin@hmc.edu
}

\author{
Halcyon Derks \\ Duke University \\ Durham, NC USA \\ hderks@math.duke.edu
}

\author{
Jennifer J. Quinn \\ University of Washington Tacoma \\ Tacoma, WA USA
}

jjquinn@uw.edu

Submitted: Mar 24, 2011; Accepted: Jun 1, 2011; Published: Jun 11, 2011

Mathematics Subject Classifications: 05A19, 11B37

\begin{abstract}
We provide two combinatorial proofs that linear recurrences with constant coefficients have a closed form based on the roots of its characteristic equation. The proofs employ sign-reversing involutions on weighted tilings.
\end{abstract}

\section{Introduction}

Given a recurrence relation and initial conditions, the goal is frequently to find a closed form expression for an arbitrary term in the sequence. While this is not always possible, the solution for homogeneous linear recurrences with constant coefficients is completely understood.

So many of our favorite number sequences, such as Fibonacci numbers and their generalizations, are precisely these. Each has beautiful tiling interpretations that make proving many identities a matter of asking a combinatorial question and answering it two different ways or describing two sets of known cardinalities and finding a correspondence between them (bijection, many-to-one mapping, almost one-to-one correspondence).

For example, the Fibonacci numbers are defined by a second order linear recurrence with coefficients of 1 and special initial conditions. More precisely, $F_{0}=0, F_{1}=1$, and for $n \geq 2, F_{n}=F_{n-1}+F_{n-2}$. For $n \geq 1, F_{n}$ is frequently interpreted as the number of tilings of a $1 \times(n-1)$-board using $1 \times 1$ squares and $1 \times 2$ dominoes [5]. Since any such tiling must end with a square or a domino, it clearly satisfies the Fibonacci recurrence and a few quick checks verify the initial conditions for $n=2$ and $n=3$ (and we happily 
declare $F_{0}=0$ and $F_{1}=1$ ). Binet's formula reveals the closed form solution for the Fibonacci numbers to be

$$
F_{n}=\frac{1}{\sqrt{5}}\left(\frac{1+\sqrt{5}}{2}\right)^{n}-\frac{1}{\sqrt{5}}\left(\frac{1-\sqrt{5}}{2}\right)^{n} .
$$

Proofs of Binet's formula range from matrix diagonalization [8] to generating functions [11] to a classic index-chasing proof by strong induction that many are familiar with from an introductory proofs class. Could there possibility be a better way? A more elegant way? A combinatorial way? In fact, a combinatorial proof involving a random tiling of an infinite board with squares and dominoes [3] can be used to explain Binet's formula and its generalization for arbitrary initial conditions. But this approach has not easily generalized to linear recurrences with constant coefficients other than 1 nor for higher order recurrences.

Here, we introduce a different combinatorial model using weighted tiles. Coupled with a sign reversing involution, Binet's formula becomes a direct consequence of counting exceptions. But better still, the weightings generalize to any linear recurrence with constant coefficients. We conclude by outlining an alternate approach to this problem using a method presaged by Zeilberger [13].

\section{Weighted Tilings to DIE for}

Given a tiling of a $1 \times n$ board (henceforth called an $n$-tiling of an $n$-board), we assign weights to individual tiles and compute the weight of the n-tiling as the product of the individual weights. For the 10-tiling illustrated in Figure 1, squares have weights of $X$, dominoes weights of $Y$, and the tiling has a weight of $X^{4} Y^{3}$.

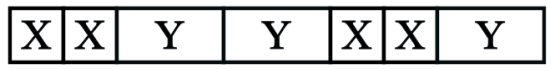

Figure 1: The weight of the illustrated 10-tiling is $X^{4} Y^{3}$.

The total weight of an $n$-board is the sum of the weights over all $n$-tilings. The total weight for a 4-board tiled with squares of weight $X$ and dominoes of weight $Y$ is $X^{4}+$ $3 X^{2} Y+Y^{2}$. Notice if all tiles have a weight of 1 then the weight of any $n$-tiling is 1 , and the total weight of an $n$-board counts all the tilings of the $n$-board.

For our weighted Fibonacci tiling, we will use several different weights for each tile type. In particular squares can have weights $\phi=\frac{1+\sqrt{5}}{2}$ or $\bar{\phi}=\frac{1-\sqrt{5}}{2}$ unless they occur as the initial tile - in which case the weight must be either $\phi / \sqrt{5}$ or $-\bar{\phi} / \sqrt{5}$. Dominoes have weight 1 except an initial domino has weight 0 . Define $W_{0}=0$. For $n \geq 1$, let $W_{n}$ be the total weight of an $n$-board under these tilings conditions. Clearly $W_{1}=\frac{1}{\sqrt{5}}(\phi-\bar{\phi})=1$ and $W_{2}=\frac{1}{\sqrt{5}}\left(\phi^{2}+\phi \bar{\phi}-\bar{\phi} \phi-\bar{\phi}^{2}+0\right)=\frac{1}{\sqrt{5}}\left(\phi^{2}-\bar{\phi}^{2}\right)=\frac{1}{\sqrt{5}}(\phi-\bar{\phi})(\phi+\bar{\phi})=1$. Requiring an initial domino to have weight 0 , we are effectively considering only those tilings that begin 
with a square. For $n>2$, we can calculate the total weight $W_{n}$ based on the weight of the last tile recursively. The contribution attributable to tilings that end with a square is $(\phi+\bar{\phi}) W_{n-1}=W_{n-1}$. Otherwise, the tiling ends in a domino and the weight contribution will be $W_{n-2}$. Thus

$$
W_{n}=W_{n-1}+W_{n-2}
$$

and our weighted tiling model matches initial conditions and recurrence relation for the Fibonacci numbers.

With this combinatorial model in hand, we can prove Binet's formula directly by creating an involution between tilings of opposite weight and determining the weight contributions of the exceptions. This technique has been coined DIE [4] for DescriptionInvolution-Exception.

\section{Proof of Binet using DIE.}

Description. Construct $n$-tilings using light squares of weight $\phi$, dark squares of weight $\bar{\phi}$, and dominoes of weight 1 , where the weights of initial light squares, dark squares, and dominoes are $\phi / \sqrt{5},-\bar{\phi} / \sqrt{5}$, and 0 respectively. We previously verified that the total weight of such $n$-tilings equals $F_{n}$.

Involution. Given an $n$-tiling, let $k$ and $k+1$ be the first cells where there is either a domino or consecutive squares of different shades (a light square followed by a dark square or vice versa.) Note $k$ ranges between 1 and $n-1$ and we will say that $k$ marks the first variation. If $k=1$ and begins with consecutive squares of different shades, switch the order of the shades and corresponding weights as illustrated in Figure 2. The weights of these two tilings are equal in magnitude but opposite in sign. So in the calculation of total weight they add to zero.

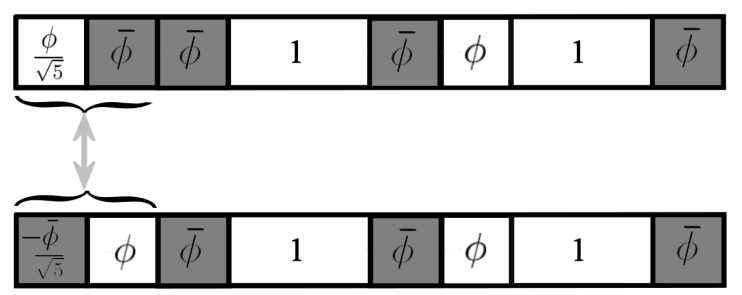

Figure 2: IF THE FIRST VARIATION OCCURS AS CONSECUTIVE SQUARES OF DIFFERENT SHADES IN POSITIONS 1 AND 2, PAIR WITH THE TILING WHERE THE FIRST TWO SQUARES HaVE OPPOSITE SHADES. ThE TILINGS PICTURED HERE HAVE WEIGHTS $\frac{1}{\sqrt{5}} \phi^{2} \bar{\phi}^{4}$ AND $-\frac{1}{\sqrt{5}} \phi^{2} \bar{\phi}^{4}-$ CONVENIENTLY ADDING TO ZERO.

If the variation occurs at $k \geq 2$ and cells $k$ and $k+1$ contain a domino, it must be preceded by squares of the same shade. Replace the domino by two squares, where the first has the same shade as the preceding squares and the second has the opposite shade as illustrated in Figure 3. Else the variation must be consecutive squares of different 
shades that are to be exchanged for a domino. Since the weight of two diverse squares is $\phi \bar{\phi}=-1$ and the weight of a domino is 1 , we are once again pairing tilings whose weights have equal magnitude but opposite sign.
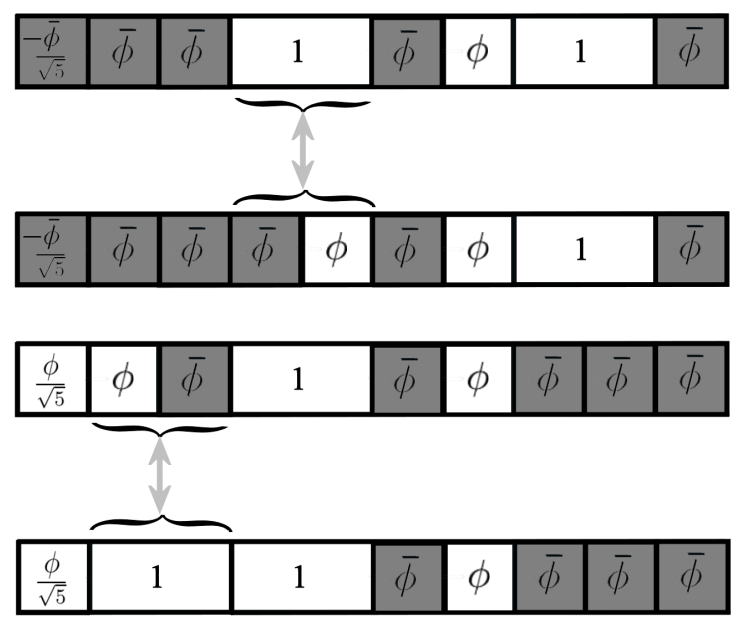

Figure 3: If first variation is Begins at CELL $k, 2 \leq k \leq n-1$.

For all $k, 1 \leq k \leq n-1$, when the mapping described above can be applied it is an involution - a second application of the mapping returns a tiling to its original configuration. Since the weights of paired tilings cancel one another, they have no effect in the calculation of total weight. Thus all that remains is to determine the weight contribution of the exceptional (unpaired) tilings.

Exception. The $n$-tilings with an initial domino or those having all the squares and no variation are unmatched by the involution. Tilings beginning with an initial domino contribute a total weight of 0 , the all-light-square tiling contributes $\frac{1}{\sqrt{5}} \phi^{n}$, and the alldark-square tiling contributes $-\frac{1}{\sqrt{5}} \bar{\phi}^{n}$. Hence the total weight of an $n$-tiling is

$$
F_{n}=\frac{1}{\sqrt{5}}\left(\phi^{n}-\bar{\phi}^{n}\right)
$$

as desired.

For those familiar with the Fibonacci numbers, it is not a surprise to see the quantities $\phi$ and $\bar{\phi}$ play a prominent role because they are the roots to $x^{2}-x-1=0$, the characteristic equation of the recurrence. This key observation motivates the weight assignments when generalizing to different coefficients and/or higher order recurrences.

\section{Characteristic Equations with Distinction}

Not all linear recurrences are created equal-some are more simply understood than others. Recall that a $k^{\text {th }}$ order homogeneous linear recurrence with constant coefficients

$$
h_{n}=a_{1} h_{n-1}+a_{2} h_{n-2}+\cdots+a_{k} h_{n-k} \quad\left(a_{k} \neq 0\right)
$$


has characteristic equation

$$
x^{k}-a_{1} x^{k-1}-a_{2} x^{k-2}-\cdots-a_{k}=0 .
$$

If equation (2) has distinct roots $r_{1}, r_{2}, \ldots, r_{k}$, then the general closed form solution to the recurrence in (1) is

$$
h_{n}=c_{1} r_{1}^{n}+c_{2} r_{2}^{n}+\cdots+c_{k} r_{k}^{n}
$$

Given any number sequence $h_{0}, h_{1}, h_{2}, \ldots$ satisfying the recurrence for $n \geq k$, there is a unique solution for coefficients $c_{1}, c_{2}, \ldots c_{k}$ so that the formula in (3) agrees with every element of the sequence including the initial conditions. Our goal is to understand the closed form solution in (3) through weighted tilings. For our example of the Fibonacci numbers, $r_{1}=\phi, r_{2}=\bar{\phi}$, and to satisfy the initial conditions $F_{0}=0$ and $F_{1}=1$ we find that $c_{1}=\frac{1}{\sqrt{5}}$ and $c_{2}=-\frac{1}{\sqrt{5}}$. We will see that the initial conditions play a critical role in determining the weights of initial tiles.

\section{Second Order}

Our first step will be to generalize the proof of Binet's formula to second order linear recurrences with arbitrary constant coefficients.

Theorem 1 Suppose the sequence $h_{0}, h_{1}, h_{2}, \ldots$ satisfies the recurrence

$$
h_{n}=a_{1} h_{n-1}+a_{2} h_{n-2}, \quad a_{2} \neq 0, \quad(n \geq 2) .
$$

If the characteristic equation $x^{2}-a_{1} x-a_{2}=0$ has distinct roots $r_{1}$ and $r_{2}$, then there exist constants $c_{1}, c_{2}$ such that

$$
h_{n}=c_{1} r_{1}^{n}+c_{2} r_{2}^{n}
$$

\section{Proof of Theorem 1 using DIE}

Description. For $n \geq 0$, let $W_{n}$ be the total weight of an $n$-board tiled with light squares, dark squares, and dominoes where the weights are specified as follows:

\begin{tabular}{|c|c|c|}
\hline Tile & \multicolumn{3}{|c|}{ Weight based on position } \\
type & initial & subsequent \\
\hline light square & $c_{1} r_{1}$ & $r_{1}$ \\
dark square & $c_{2} r_{2}$ & $r_{2}$ \\
domino & $-\left(c_{1}+c_{2}\right) r_{1} r_{2}$ & $-r_{1} r_{2}$ \\
\hline
\end{tabular}

Here $c_{1}$ and $c_{2}$ are variables to be determined after finding the general form of the solution. We define the empty tiling to have weight $W_{0}=c_{1}+c_{2}$.

Verifying the Recurrence. We partition $W_{n}$ based on the weight of the last tile. When $n>2$, the board is long enough to prevent the last tile from also playing the role of an 
initial tile. Tilings that end in a light square contribute a weight of $r_{1} W_{n-1}$, that end in a dark square contribute $r_{2} W_{n-1}$, and that end in a domino contribute $-r_{1} r_{2} W_{n-2}$. By similar reasoning and our choice of $W_{0}$, the recurrence also works when $n=2$. Thus $W_{n}=\left(r_{1}+r_{2}\right) W_{n-1}-r_{1} r_{2} W_{n-2}$. But $r_{1}$ and $r_{2}$ are roots of the characteristic polynomial $x^{2}-a_{1} x-a_{2}=\left(x-r_{1}\right)\left(x-r_{2}\right)$. Hence $r_{1}+r_{2}=a_{1}$ and $r_{1} r_{2}=-a_{2}$ and we see that $W_{n}$ satisfies the same recurrence as $h_{n}, W_{n}=a_{1} W_{n-1}+a_{2} W_{n-2}$.

Involution. Given an $n$-tiling $(n \geq 1)$, let $k$ mark the first variation. When $k \geq 2$, exchange a domino of weight $-r_{1} r_{2}$ for consecutive squares of different shades (weight $r_{1} r_{2}$ ) and vice versa. Remember when replacing a domino, the shade of the $k^{\text {th }}$ cell must agree with the shade of the $(k-1)^{s t}$ cell. See Figure 4 . The paired tilings have weights of equal magnitude but opposite sign.

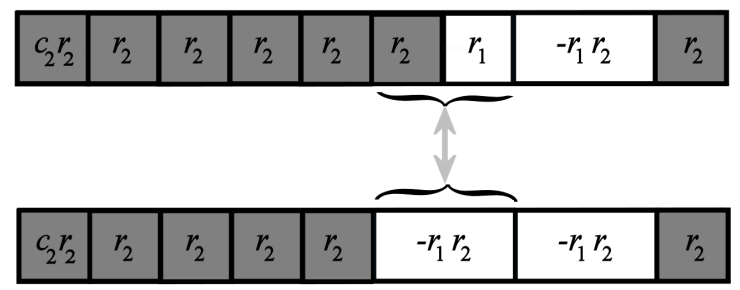

Figure 4: IF VARIATION BEgins AT CELL $k, 2 \leq k \leq n-1$.

Exceptions. For $n \geq 1$, the unmatched $n$-tilings are the all-square tilings of the same shade or those beginning with a variation. Fortunately we can form groups of $n$-tilings with initial variations to take advantage of further cancellation. An $n$-tiling with an initial variation begins in one of three ways: light square followed by a dark square (weight $c_{1} r_{1} r_{2}$ ), dark square followed by a light square (weight $c_{2} r_{2} r_{1}$ ), or a domino (weight $\left.-\left(c_{1}+c_{2}\right) r_{1} r_{2}\right)$. Clearly any exceptional tiling beginning with a variation can be grouped with the two alternate beginnings to create a 3 -set of $n$-tilings whose weights sum to zero. See Figure 5. Consequently the only exceptional $n$-tilings contributing to the total weight

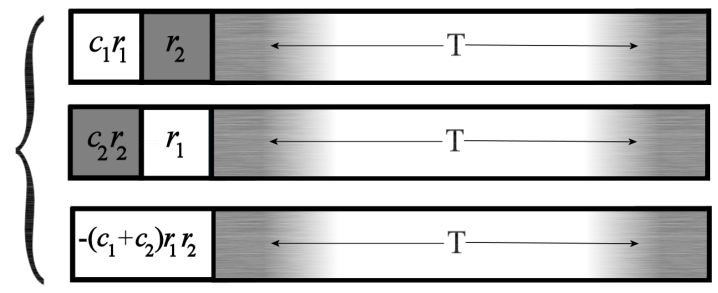

Figure 5: If EXCEPTION BEGINS AT CELL 1, FORM 3 -SETS OF $n$-Tilings $(n \geq 2)$ TO CREATE A GROUPING OF NET WEIGHT ZERO.

are the all-square tilings of the same shade. Thus the general solution to the recurrence is

$$
h_{n}=W_{n}=c_{1} r_{1}^{n}+c_{2} r_{2}^{n}
$$


for $n \geq 0$. (The $n=0$ case follows from our definition of $W_{0}$.) To find specific values of the variables $c_{1}$ and $c_{2}$ so that the general solution matches the initial conditions of the sequence, we need to solve the linear system

$$
\begin{aligned}
c_{1}+c_{2} & =h_{0} \\
r_{1} c_{1}+r_{2} c_{2} & =h_{1} .
\end{aligned}
$$

The coefficient matrix $\left[\begin{array}{ll}1 & 1 \\ r_{1} & r_{2}\end{array}\right]$ has determinant equal to $r_{2}-r_{1}$. Since $r_{1}$ is distinct from $r_{2}$, the determinant is nonzero and the system has a unique solution. Thus the closed form solution $h_{n}=c_{1} r_{1}^{n}+c_{2} r_{2}^{n}$ holds for $n \geq 0$.

\section{Higher Order}

Proceeding to a higher order linear recurrence will require longer tiles and more weights. We call a $1 \times t$ tile a $t$-omino; linear recurrence relations of order $k$ will require tiles of all lengths from squares to $k$-ominoes. As with the second order recurrence, we start with the situation where the characteristic equation has distinct roots. The weights of squares will be selected from the roots of the characteristic equation and weights of $t$-ominoes will be a signed product of $t$ distinct roots. Tiles of odd length will be weighted positively and tiles of even length, negatively. Weights of the first tiles will follow these general rules but be multiplied by an appropriate factor to ensure the total weight of an $n$-tiling can be chosen to match the given initial conditions for $0 \leq n \leq k-1$. We must broaden our idea of a variation in this context. It is still meant to indicate the involvement of two distinct roots in the weights. But this can happen in one of two ways:

1. a tile of length 2 or greater marks a variation since the weight of this tile includes at least two distinct roots;

2. a square of weight $r_{i}$ marks a variation only if the subsequent tile (of any length) does not include the weight $r_{i}$ as a factor.

In Figure 6, the second, fifth, sixth, and seventh tiles (beginning on cells 2, 5, 9, and 10 respectively) mark variations. The square on cell 4 is not a variation since it's weight of $r_{2}$ occurs in the subsequent 4-omino.

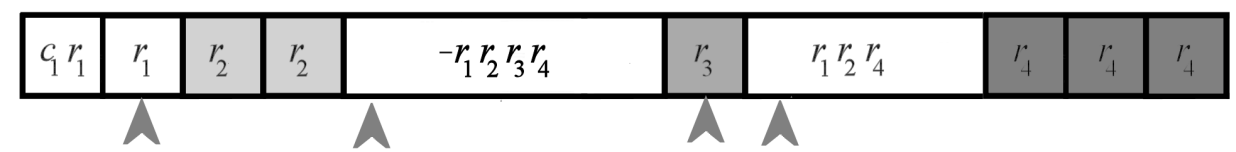

Figure 6: Weighted tiling With squares, 3-OMinoes, 4-OMinoes Showing loCATION OF VARIATIONS. 
Theorem 2 Suppose the sequence $h_{0}, h_{1}, h_{2}, \ldots$ satisfies the recurrence

$$
h_{n}=a_{1} h_{n-1}+a_{2} h_{n-2}+\cdots+a_{k} h_{n-k} \quad a_{k} \neq 0, \quad(n \geq k) .
$$

If the characteristic equation $x^{k}-a_{1} x^{k-1}-a_{2} x^{k-2}-\cdots-a_{k}=0$ has distinct roots $r_{1}, r_{2}, \ldots, r_{k}$, then there exist constants $c_{1}, c_{2}, \ldots, c_{k}$ such that

$$
h_{n}=c_{1} r_{1}^{n}+c_{2} r_{2}^{n}+\cdots+c_{k} r_{k}^{n}
$$

Once again, we will first find the general solution to the recurrence and then show how it specializes to a particular solution to match the given initial conditions of a sequence.

\section{Proof of Theorem 2 using DIE}

Description. Let $W_{n}$ be the total weight of an $n$-board tiled with squares, dominoes, $\ldots, k$-ominoes. We define $W_{0}=c_{1}+c_{2}+\cdots+c_{k}$ and for $n \geq 1$, the weight of an $n$-tiling

\begin{tabular}{|c|c|}
\hline $\begin{array}{l}\text { Tile } \\
\text { type }\end{array}$ & $\begin{array}{l}\text { Available weights } \\
\text { for initial tiles }\end{array}$ \\
\hline square & for $i=1,2, \ldots, k$ \\
\hline domino & for $1 \leq i<j \leq k$ \\
\hline $\begin{array}{c}: \\
t \text {-omino }\end{array}$ & $\begin{array}{c}\vdots \\
(-1)^{t+1}\left(c_{i_{1}}+c_{i_{2}}+\cdots+c_{i_{+}}\right) r_{i_{1}} r_{i_{2}} \cdots r_{i_{+}} \text {for } 1 \leq i_{1}<i_{2}<\ldots<i_{t} \leq k\end{array}$ \\
\hline : & : \\
\hline$k$-omino & $(-1)^{k+1}\left(c_{1}+c_{2}+\cdots+c_{k}\right) r_{1} r_{2} \cdots r_{k}$ \\
\hline
\end{tabular}
is the product of the weights of its tiles, defined as follows:

where $c_{1}, c_{2}, \ldots, c_{k}$ are variables to be determined once the general solution is found.

\begin{tabular}{|c|cc|}
\hline $\begin{array}{c}\text { Tile } \\
\text { type }\end{array}$ & \multicolumn{2}{|c|}{$\begin{array}{c}\text { Available weights } \\
\text { for subsequent tiles }\end{array}$} \\
\hline square & $r_{i}$ & for $i=1,2, \ldots, k$ \\
domino & $-r_{i} r_{j}$ & for $1 \leq i<j \leq k$ \\
$\vdots$ & $\vdots$ & \\
$t$-omino & $(-1)^{t+1} r_{i_{1}} r_{i_{2}} \cdots r_{i_{t}}$ & for $1 \leq i_{1}<i_{2}<\ldots<i_{t} \leq k$ \\
$\vdots$ & $\vdots$ & \\
$k$-omino & $(-1)^{k+1} r_{1} r_{2} \cdots r_{k}$ \\
\hline
\end{tabular}

Notice that the weight of a $t$-omino (for $1 \leq t \leq k$ ) contains the product of $t$ distinct roots. So there are $\left(\begin{array}{l}k \\ t\end{array}\right)$ different weights that can be assigned regardless of whether it occurs in initial position or not. 
Verifying the Recurrence. For $n \geq k$, we partition $W_{n}$ based on the length of the last tile. It is important to remember the relationship between the roots of a characteristic equation and its coefficients. When

$$
x^{k}-a_{1} x^{k-1}-a_{2} x^{k-2}-\cdots-a_{k}=\left(x-r_{1}\right)\left(x-r_{2}\right) \cdots\left(x-r_{k}\right),
$$

the coefficient of $x^{k-t}, 1 \leq t \leq k$, is

$$
-a_{t}=\sum_{\substack{S \subset\{1, \ldots, k\} \\|S|=t}} \prod_{s \in S}-r_{s} .
$$

Said another way,

$$
a_{t}=\sum_{1 \leq i_{1}<i_{2}<\cdots<i_{t} \leq k}(-1)^{t+1} r_{i_{1}} r_{i_{2}} \cdots r_{i_{t}}
$$

or $a_{t}$ represents the sum over all possible $t$-omino weights. Thus the weight contribution for $n$-tilings that end in a $t$-omino, is $a_{t} W_{n-t}$. Summing over all possible tile lengths gives the desired recurrence $W_{n}=a_{1} W_{n-1}+a_{2} W_{n-2}+\cdots+a_{k} W_{n-k}$.

Involution. Given an $n$-tiling, let $k$ mark the first variation. For $k \geq 2$, exchange a square of weight $r_{j}$ followed by a $t$-omino of weight $(-1)^{t+1} r_{i_{1}} r_{i_{2}} \cdots r_{i_{t}}$ by a $(t+1)$-omino of weight $(-1)^{t+2} r_{j} r_{i_{1}} r_{i_{2}} \cdots r_{i_{t}}$. Otherwise the variation marks a $t$-omino that is to be replaced by a square and a $(t-1)$-omino, where the weight given to the square on the $k^{t h}$ cell agrees with the weight of the square on cell $(k-1)$. It is not possible for a variation to mark a square preceding a $k$-omino, since all roots occur in the weight of the largest tile. There is never a question of creating a tile too long for our consideration. See Figure 7. The paired tilings have weights of equal magnitude but opposite sign.

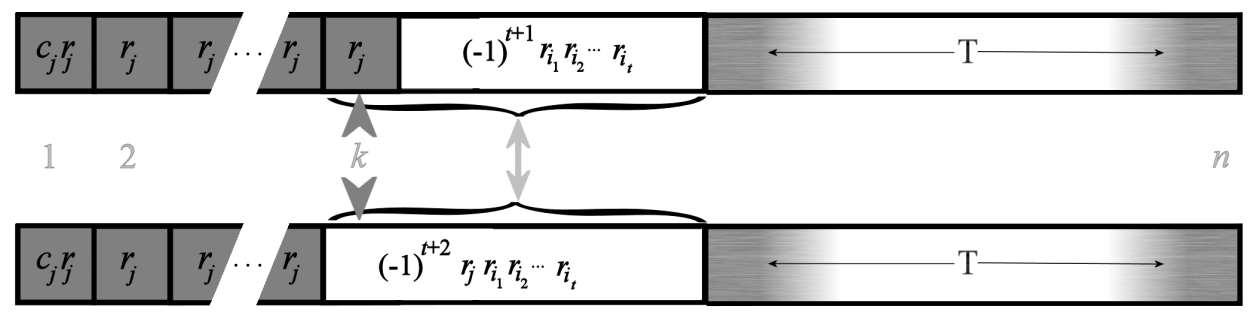

Figure 7: If VARIATION BEGINS AT CELL $k, 2 \leq k \leq n-1$.

Exceptions. The unmatched $n$-tilings are the all-square tilings without variation or those beginning with a variation. Fortunately we can again form groups of $n$-tilings with initial variations to take advantage of further cancellation. Suppose an $n$-tiling begins with a $t$-omino $(t \geq 2)$ of weight $(-1)^{t+1}\left(c_{i_{1}}+c_{i_{2}}+\cdots+c_{i_{t}}\right) r_{i_{1}} r_{i_{2}} \cdots r_{i_{t}}$. Group this $n$-tiling with $t$ others - specfically the ones beginning with a square of weight $c_{i_{q}} r_{i_{q}}$ and a $(t-1)$-omino of weight $(-1)^{t} r_{i_{1}} r_{i_{2}} \cdots r_{i_{t}} / r_{i_{q}}$ for $q=1,2, \ldots, t$. The net weight contribution of these $t+1 n$-tilings is zero. Thus $n$-tilings that begin with a variation can partitioned into sets whose net weight contribution is zero. Consequently the only exceptional $n$-tilings 
contributing to the total weight are the all-square tilings of the same weight. Thus, for $n \geq 0$,

$$
W_{n}=c_{1} r_{1}^{n}+c_{2} r_{2}^{n}+\cdots+c_{k} r_{k}^{n}
$$

Notice that the computation of the total weight was independent of the length of the tiling. So the involution and exception analysis also holds for $n \geq 1$.

To find specific values of the variables $c_{1}, c_{2}, \ldots, c_{k}$, in agreement with the initial conditions of the sequence, we need to solve the linear system

$$
\begin{aligned}
& c_{1}+c_{2}+\cdots+\quad c_{k}=h_{0} \\
& r_{1} c_{1}+r_{2} c_{2}+\cdots+r_{k} c_{k}=h_{1} \\
& r_{1}^{2} c_{1}+r_{2}^{2} c_{2}+\cdots+r_{k}^{2} c_{k}=h_{2} \\
& r_{1}^{k-1} c_{1}+r_{2}^{k-1} c_{2}+\cdots+r_{k}^{k-1} c_{k}=h_{k-1} \text {. }
\end{aligned}
$$

The coefficient matrix is Vandermonde and its determinant is $\prod_{1 \leq i<j \leq k}\left(r_{j}-r_{i}\right)$. This classic result has many beautiful proofs (see e.g. [1, 6, 9]), including combinatorial ones $[2,7,10]$. Distinct roots guarantee a nonzero determinant and the existence of a unique solution for $c_{1}, c_{2}, \ldots, c_{k}$ for any choice of initial conditions. Thus the closed form solution $h_{n}=W_{n}=c_{1} r_{1}^{n}+c_{2} r_{2}^{n}+\cdots+c_{k} r_{k}^{n}$ holds for $n \geq 0$.

\section{Characteristic Equations with Repetition}

To extend our weighted tiling approach to linear recurrences whose characteristic equation has repeated roots, we are going to introduce coins to the weighted tilings. We begin with a simpler situation of a single root of high multiplicity before proceeding to the most general situation.

Theorem 3 Suppose the sequence $h_{0}, h_{1}, h_{2}, \ldots$ satisfies the recurrence

$$
h_{n}=a_{1} h_{n-1}+a_{2} h_{n-2}+\cdots+a_{k} h_{n-k} \quad a_{k} \neq 0, \quad(n \geq k) .
$$

If the characteristic polynomial factors as $(x-r)^{k}$, then there exist constants $c_{1}, c_{2}, \ldots, c_{k}$ such that

$$
h_{n}=c_{1} r^{n}+c_{2} n r^{n}+\cdots+c_{k} n^{k-1} r^{n} .
$$

Begin by thinking of the $k$ roots as distinct $r_{1}, r_{2}, \ldots, r_{k}$ (the first root, the second root, third root, etc.) and use them to assign weights to tiles as was previously done. Of course numerically $r_{1}=r_{2}=\cdots=r_{k}=r$. If you prefer, you can think of a square of weight $r_{1}$ as white, a square of weight $r_{k}$ as black, and squares of weights in between as proportionally darker shades of grey. For a given weighted tiling, if $r_{m}$ is the largest root that appears (meaning $m$ is the largest index involved in any tile weight), then we place 


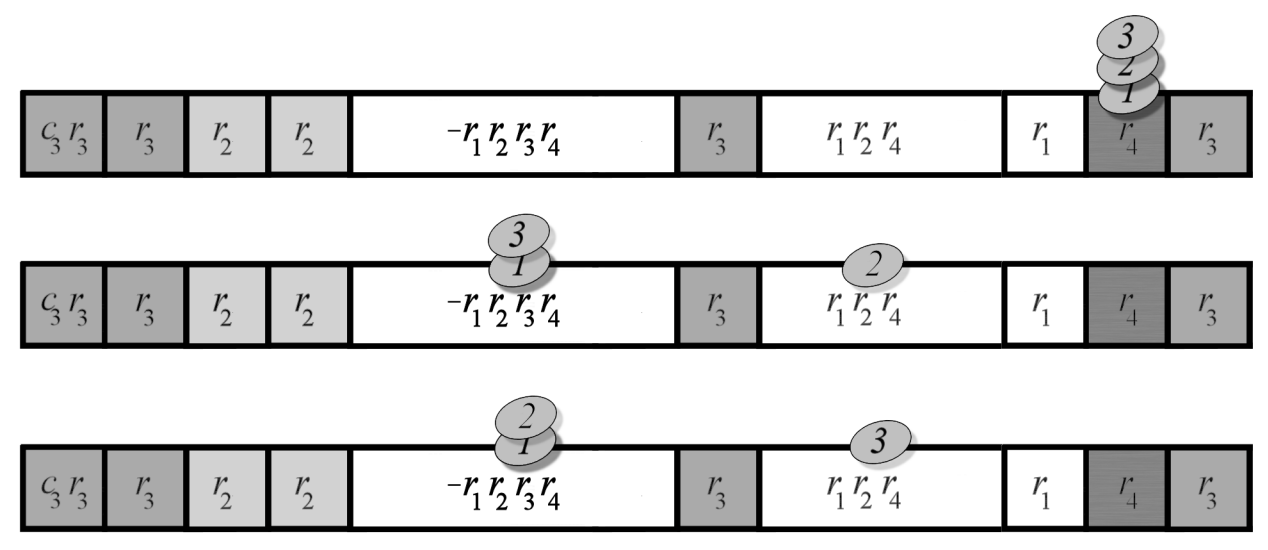

Figure 8: EXAMPles OF 15-TILINGS With LARGEST REPEATED ROOT $r_{4}$ REQUIRING 3 DifFERENT COINS. Notice that THE SECOND AND THIRD EXAMPles ARE CONSIDERED DIFFERENT BECAUSE THE COINS ARE DISTINCT.

$m-1$ distinct coins on the tiling where a coin can only be placed on a tile whose weight contains $r_{m}$ as a factor. See examples in Figure 8.

Description. Define $W_{0}=c_{1}$ and for $n \geq 1$ let $W_{n}$ be the total weight of a coined $n$-board tiled with squares, dominoes, $\ldots, k$-ominoes where the weights are specified as follows:

\begin{tabular}{|c|c|}
\hline $\begin{array}{l}\text { Tile } \\
\text { type }\end{array}$ & $\begin{array}{l}\text { Available weights } \\
\text { for initial tiles }\end{array}$ \\
\hline $\begin{array}{l}\text { square } \\
\text { domino }\end{array}$ & $\begin{array}{cc}c_{i} r_{i} & \text { for } i=1,2, \ldots, k \\
-\left(c_{i}+c_{j}\right) r_{i} r_{j} & \text { for } 1 \leq i<j \leq k\end{array}$ \\
\hline $\begin{array}{c}\vdots \\
t \text {-omino }\end{array}$ & $\begin{array}{c}\vdots \\
(-1)^{t+1}\left(c_{i_{1}}+c_{i_{2}}+\cdots+c_{i_{t}}\right) r_{i_{1}} r_{i_{2}} \cdots r_{i_{t}} \quad \text { for } 1 \leq i_{1}<i_{2}<\ldots<i_{t} \leq k \\
:\end{array}$ \\
\hline$k$-omino & $(-1)^{k+1}\left(c_{1}+c_{2}+\cdots+c_{k}\right) r_{1} r_{2} \cdots r_{k}$ \\
\hline
\end{tabular}

where $c_{1}, c_{2}, \ldots, c_{k}$ are variables to be determined once the general solution is found.

\begin{tabular}{|c|cc|}
\hline $\begin{array}{c}\text { Tile } \\
\text { type }\end{array}$ & $\begin{array}{c}\text { Available weights } \\
\text { for subsequent tiles }\end{array}$ \\
\hline square & $r_{i}$ & for $i=1,2, \ldots, k$ \\
domino & $-r_{i} r_{j}$ & for $1 \leq i<j \leq k$ \\
$\vdots$ & $\vdots$ & \\
$t$-omino & $(-1)^{t+1} r_{i_{1}} r_{i_{2}} \cdots r_{i_{t}}$ & for $1 \leq i_{1}<i_{2}<\ldots<i_{t} \leq k$ \\
$\vdots$ & $\vdots$ & \\
$k$-omino & $(-1)^{k+1} r_{1} r_{2} \cdots r_{k}$ \\
\hline
\end{tabular}

For a given tiling, if $m$ is the largest index involved in any tile weight then place $m-1$ distinct coins on the tiling; coins may be placed only on tiles with weights containing $r_{m}$ as a factor. 
Verifying the Recurrence. For $n \geq k$, we partition $W_{n}$ based on the number of coins on the last tile. We will show that the total weight contributed by $n$-tilings with at least one coin on the last tile is zero. The important tilings are those with uncoined final tiles and these will be counted based on the weight and length of the last tile.

Consider the weight contribution of $n$-tilings with largest root $r_{m}$ having $q$ coins on the last tile, $1 \leq q \leq m-1,2 \leq m \leq k$. Since the last tile has at least one coin, $r_{m}$ must be a factor of its weight. Further, no tile can be longer than $m$ or else a larger root would be involved. We pair tilings based on the last variation: a tile of length greater than or equal to 2 that contains $r_{m}$ as a weight where the only tiles that follow it (if any) are squares of weight $r_{m}$, or it is a tile of length greater than or equal to 1 that does not contain $r_{m}$, where the only tiles that follows (at least one) are squares of weight $r_{m}$. See Figure 9 . As

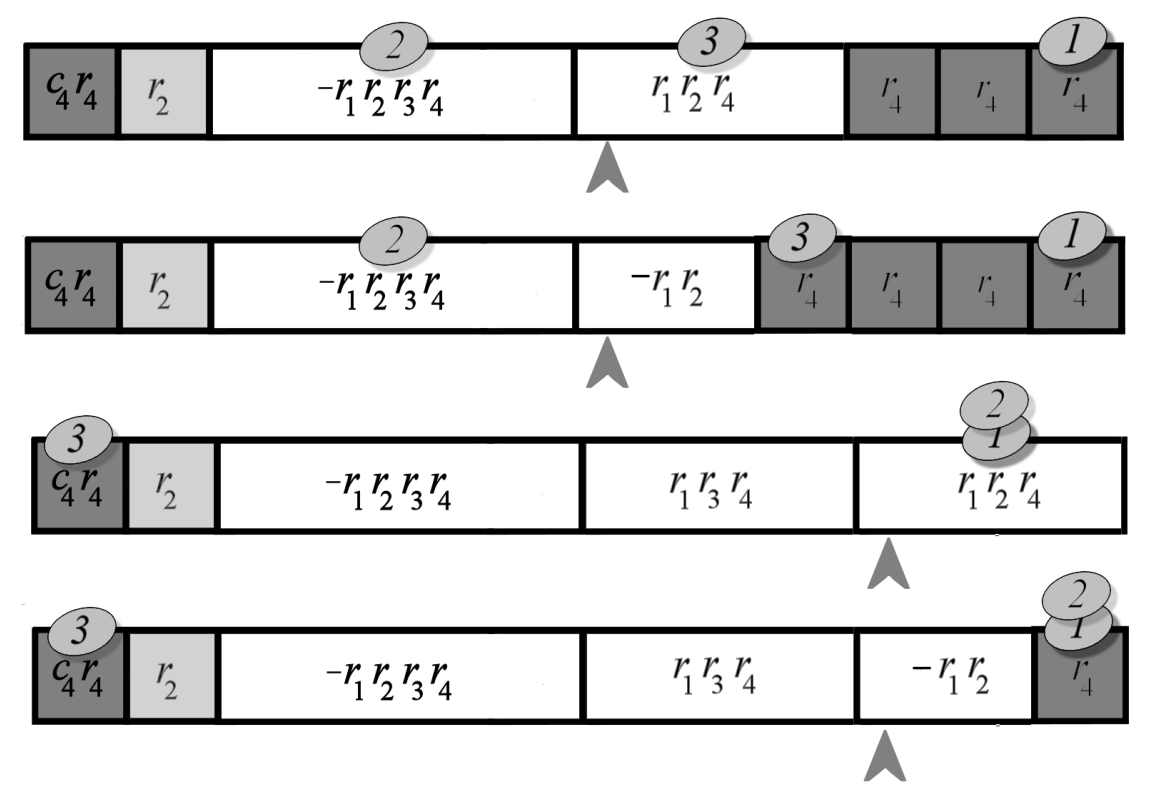

Figure 9: Coined And Weighted 12-tilings Where the LASt Tile includes A ROOT OF LARGEST WEIGHT AND AT LEAST ONE COIN. THE last variation FOR EACH EXAMPLE IS MARKED BY A GREY ARROW.

long as the variations do not involve the first tile of the $n$-board, switching between the two types of variations maintains the magnitude of the tilings' weights but changes the sign. Furthermore, if coins are involved in the variation, they follow the tile with factor $r_{m}$. The total number of coins remains constant at $m-1$ and the last tile maintains $q$ coins. The unpaired tilings in this subset are those whose last variation involves the first tile (specifically the first tile has length $m$ or less with weights selected from $r_{1}, r_{2}, \ldots r_{m}$ followed by all squares of weight $r_{m}$ ) or the coined all-square tilings of weight $c_{m} r_{m}^{n}$. The total weight of these exceptions is

$$
\sum_{t=1}^{m} \sum_{1 \leq i_{1}<i_{2}<\cdots<i_{t} \leq m}(-1)^{t+1}\left(c_{i_{1}}+c_{i_{2}}+\cdots+c_{i_{t}}\right) \underbrace{r_{i_{1}} r_{i_{2}} \cdots r_{i_{t}} r_{m}^{n-t}}_{r^{n}} .
$$


The analysis of this sum depends only on the interactions of the special coefficient $c_{1}, c_{2}, \ldots, c_{m}$ since every term has a common factor of $r^{n}$. For $1 \leq j \leq m, c_{j}$ is either added or subtracted depending on whether $r_{j}$ is a factor of an initial tile of odd or even length. Since $r_{j}$ occurs as a factor of an initial square $\left(\begin{array}{c}m-1 \\ 0\end{array}\right)$ times, a factor of an initial domino $\left(\begin{array}{c}m-1 \\ 1\end{array}\right)$ times, an initial tromino $\left(\begin{array}{c}m-1 \\ 2\end{array}\right)$, and an initial $m$-omino $\left(\begin{array}{l}m-1 \\ m-1\end{array}\right)$ times, the contribution of the initial weighting factor $c_{j}$ in (4) is the alternating sum $\left(\begin{array}{c}m-1 \\ 0\end{array}\right)-\left(\begin{array}{c}m-1 \\ 1\end{array}\right)+\left(\begin{array}{c}m-1 \\ 2\end{array}\right)+\cdots+(-1)^{m-1}\left(\begin{array}{c}m-1 \\ m-1\end{array}\right)=0$. Summing over all possible values of $q$ and $m$, we conclude that the total weight contribution for $n$-tilings with at least one coin on the last tile is zero.

It remains to determine the weight contribution of the coined $n$-tilings with uncoined final tiles. The target recurrence

$$
a_{1} W_{n-1}+a_{2} W_{n-2}+\cdots+a_{k} W_{n-k} \quad a_{k} \neq 0, \quad(n \geq k)
$$

is achieved by naively appending uncoined weighted $t$-ominoes to properly weighted and coined $(n-t)$-tilings for $t=1,2, \ldots k$. This procedure is a tad overzealous because it introduces some improper coined tilings. If the newly appended tile includes larger roots than previously occurred, the number and placement of coins is no longer valid. For example, appending an uncoined square of weight $r_{5}$ to any tiling in Figure 8 or an uncoined 4-omino of weight $-r_{2} r_{3} r_{4} r_{5}$ to any tiling in Figure 9 creates improper 16-tilings as the largest root is $r_{5}$ and the tiling now requires 4 coins distributed to tiles containing a factor of $r_{5}$.

Fortunately, we can show how the invalid coined $n$-tilings created by this process contribute a net weight of zero. Suppose that attaching an uncoined $t$-omino $(1 \leq t \leq k)$ to an $(n-t)$-tiling creates an invalid $n$-tiling. Then the $t$-omino must have at least one root greater than $r_{m}$, where $r_{m}$ is the largest root that appears in the valid $(n-t)$-tiling. Suppose exactly $j$ of the roots in the $t$-omino are greater than $r_{m}$, where $1 \leq j \leq t$. We now consider two cases, depending on whether $j \leq t-1$ or $j=t$. If $j \leq t-1$, then we split the $t$-omino into a $j$-omino with the $j$ largest roots preceded by an uncoined $(t-j)$-omino with $t-j$ roots that are no bigger than $r_{m}$. This is an invalid $n$-tiling that arises from appending a $j$-omino (with all roots greater than $r_{m}$ ) to a valid $(n-j$ )-tiling that ends with an uncoined tile. Since the sign of the $t$-omino is $(-1)^{t-1}$ and the sign of the split tiles is $(-1)^{j-1}(-1)^{t-j-1}=(-1)^{t-2}$, the pair of invalid tilings are of opposite sign. On the other hand, if $j=t$ (all roots of the $t$-omino are greater than $r_{m}$ ), then we merge the $t$-omino with the preceding tile (say of length $i \geq 1$ ), provided that the $i$-omino is uncoined. This creates an invalid, opposite signed, $n$-tiling that ends with an uncoined $(t+i)$-omino, preceded by a valid coined $(n-t-i)$-tiling, (where $t$ of the roots of the $(t+i)$-omino are greater than $\left.r_{m}\right)$. Note that since the roots on the $t$-omino and $i$-omino are distinct, then the $(t+i)$-omino has length at most $k$. Further, if $n \geq k+1$, the $i$-omino could not have been the initial tile. If $n=k$, the only way that the uncoined $i$-omino can be the initial tile, is if the $i$-onimo is a square of weight $c_{1} r_{1}$ (otherwise it would have a coin), and the $t$-omino is a $(k-1)$-omino of weight $(-1)^{k-2} r_{2} \ldots r_{k}$. But this is cancelled out by the $a_{k} W_{0}$ term which contributes $(-1)^{k-1} r_{1} r_{2} \ldots r_{k} c_{1}$, by our choice of $W_{0}$. 
The only remaining invalid $n$-tilings are those consisting of a valid coined $(n-t)$-tiling with maximum root $r_{m}$ followed by a $t$-omino (of weight $r^{t}$ ) where all $t$ roots are greater than $r_{m}$, and the $(n-t)$-tiling ends with a coined tile. As we saw earlier, the total weight contribution for coined tilings (this time $(n-t)$-tilings) that end with a coined tile is zero. Hence only valid coined tilings are making net contributions in the desired recurrence, so we conclude that for $n \geq k, W_{n}=a_{1} W_{n-1}+a_{2} W_{n-2}+\cdots+a_{k} W_{n-k}$.

Involution. Given a coined $n$-tiling, let $\ell$ mark the first cell of the first variation. For $\ell \geq 2$, exchange a square of weight $r_{j}$ followed by a $t$-omino of weight $(-1)^{t+1} r_{i_{1}} r_{i_{2}} \cdots r_{i_{t}}$ by a $(t+1)$-omino of weight $(-1)^{t+2} r_{j} r_{i_{1}} r_{i_{2}} \cdots r_{i_{t}}$. Otherwise the variation marks a $t$ omino that is to be replaced by a square and a $(t-1)$-omino, where the weight given to the square on the $\ell^{\text {th }}$ cell agrees with the weight of the square on cell $(\ell-1)$. Coins follow the maximum root. It is not possible for a variation to mark a square preceding a $k$-omino, since all roots occur in the weight of the largest tile, so there is never a question of creating a tile too long for our consideration. The paired coined $n$-tilings have weights of equal magnitude but opposite sign.

Exceptions. The unmatched $n$-tilings are the all-square coined tilings without variation or those beginning with a variation. Groups of $n$-tilings with initial variations, taking coins into account, will again sum to zero. Suppose an $n$-tiling begins with a $t$-omino $(t \geq 2)$ of weight $(-1)^{t+1}\left(c_{i_{1}}+c_{i_{2}}+\cdots+c_{i_{t}}\right) r_{i_{1}} r_{i_{2}} \cdots r_{i_{t}}$. Group this $n$-tiling with $t$ others - specially the ones beginning with a square of weight $c_{i_{q}} r_{i_{q}}$ and a $(t-1)$-omino of weight $(-1)^{t} r_{i_{1}} r_{i_{2}} \cdots r_{i_{t}} / r_{i_{q}}$ for $q=1,2, \ldots, t$. The net weight contribution of these $t+1$ $n$-tilings is zero. Thus $n$-tilings that begin with a variation can be partitioned into sets whose net weight contribution is zero. Consequently the only exceptional coined $n$-tilings contributing to the total weight are the all-square coined tilings of the same weight. For $1 \leq j \leq k$, the all square tiling of weight $c_{j} r_{j}^{n}$ requires $j-1$ distinct coins and consequently contributes $c_{j} n^{j-1} r_{j}^{n}=c_{j} n^{j-1} r^{n}$. Summing over all $j$ gives

$$
W_{n}=c_{1} r^{n}+c_{2} n r^{n}+\cdots+c_{k} n^{k-1} r^{n} .
$$

The computation of the total weight was independent of the length of the tiling. So the involution and exception analysis also holds for $n \geq 1$.

To find specific values of the variables $c_{1}, c_{2}, \ldots, c_{k}$, so that the general solution matches the initial conditions of the sequence, we need to solve the linear system

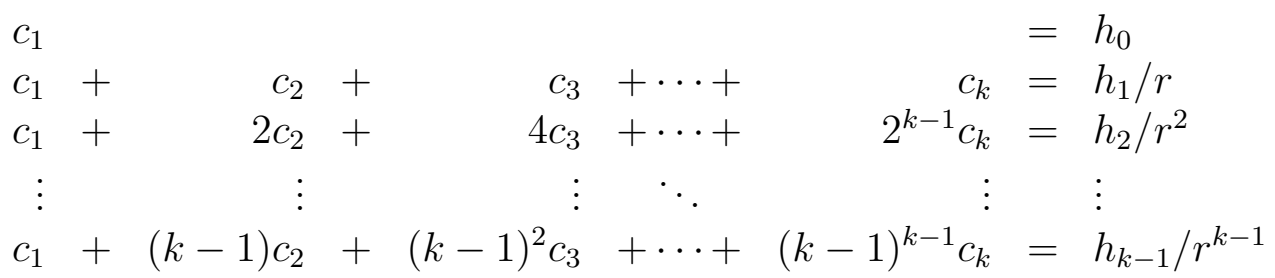

The coefficient matrix is again Vandermonde, guaranteeing a nonzero determinant and the existence of a unique solution for $c_{1}, c_{2}, \ldots, c_{k}$ for any choice of initial conditions. Thus the closed form solution $h_{n}=c_{1} r^{n}+c_{2} n r^{n}+\cdots+c_{k} n^{k-1} r^{n}$ holds for $n \geq 0$. 


\section{In Greatest Generality}

No new ideas are required to complete the discussion to all homogeneous linear recurrences with constant coefficients.

Theorem 4 Suppose the sequence $h_{0}, h_{1}, h_{2}, \ldots$ satisfies the recurrence

$$
h_{n}=a_{1} h_{n-1}+a_{2} h_{n-2}+\cdots+a_{k} h_{n-k} \quad a_{k} \neq 0, \quad(n \geq k) .
$$

If the characteristic polynomial has distinct roots $r_{1}, r_{2}, \ldots, r_{t}$ of multiplicities $m_{1}, m_{2}, \ldots$, $m_{t}$ respectively, then there exist $k$ constants $c_{(1,1)}, c_{(1,2)}, \ldots, c_{\left(1, m_{1}\right)}, c_{(2,1)}, c_{(2,2)}, \ldots, c_{\left(2, m_{2}\right)}$, $\ldots, c_{(t, 1)}, \ldots, c_{\left(t, m_{t}\right)}$, such that

$$
h_{n}=\sum_{i=1}^{t}\left(c_{(i, 1)}+c_{(i, 2)} n+\cdots+c_{\left(i, m_{i}\right)} n^{m_{i}-1}\right) r_{i}^{n} .
$$

Notice if all the roots of the characteristic polynomial are distinct, this reduces the Theorem 2 and if the roots are all the same, it reduces to Theorem 3. As before, we think of the $k$ roots as distinct and use them to assign weights to tiles. Given a weighted tiling, assign coins for each (truly) distinct root based on the largest multiplicity of the root occurring as a weight. Say if the first, second, and fourth copies of $r_{1}$ occur as weighting factors, we must distribute 3 coins among the tiles containing the fourth copy of $r_{1}$ as a factor. If only the second copy of $r_{3}$ occurs, we place 1 coin on some tile containing that factor of $r_{3}$ and so on. To verify the recurrence for $n>k$, partition the tilings based on the number of coins on the last tile and show that the total weight contributed by $n$-tilings with at least one coin on the last tile is zero. The target recurrence includes improperly coined tilings that make a net contribution of zero to the total weight as before. The involution is based on the first variation and the analysis of exceptions remain the same. The formula is valid for $n \geq 1$ and linear algebra guarantees that a unique solution for the coefficients $c_{(1,1)}, c_{(1,2)}, \ldots, c_{\left(1, m_{1}\right)}, c_{(2,1)}, c_{(2,2)}, \ldots, c_{\left(2, m_{2}\right)}, \ldots, c_{(t, 1)}, \ldots, c_{\left(t, m_{t}\right)}$ exists.

\section{$5 \quad$ An alternative approach}

There is another way to approach this problem using sign-reversing involutions, without using DIE, since there will be no exceptions. Instead of interpreting $h_{n}$ as counting weighted tilings and then canceling to get the closed form, we reverse the process to show that the closed form satisfies the recurrence. For example, for the second order case with distinct roots, to show that $c_{1} r_{1}^{n}+c_{2} r_{2}^{n}$ satisfies the recurrence, it suffices (by linearity and symmetry) to show that $h_{n}=r_{1}^{n}$ satisfies $h_{n}=a_{1} h_{n-1}+a_{2} h_{n-2}$ where $a_{1}=\left(r_{1}+r_{2}\right)$ and $a_{2}=-r_{1} r_{2}$. That is, we need to show, for $n \geq 2$,

$$
r_{1}^{n}-\left(r_{1}+r_{2}\right) r_{1}^{n-1}+r_{1} r_{2} r_{1}^{n-2}=0 .
$$

More generally, for the higher order recurrence with $k$ distinct roots, the identity to be proved is, for $n \geq k$,

$$
r_{1}^{n}-e_{1} r_{1}^{n-1}+e_{2} r_{1}^{n-2}-\cdots+(-1)^{k} e_{k} r_{1}^{n-k}=0,
$$


where $e_{t}=\sum_{1 \leq i_{1}<\cdots<i_{t} \leq k} r_{i_{1}} \cdots r_{i_{t}}$.

Here, our tilings consist of a single $t$-omino (for some $0 \leq t \leq k$ ) followed by $n-t$ squares of weight $r_{1}$. In this model, for $t \geq 1$, the weight of an initial $t$-omino with label $r_{i_{1}} \cdots r_{i_{t}}$ is $(-1)^{t} r_{i_{1}} \cdots r_{i_{t}}$, and the weight of a tiling is the product of the weights of its tiles. The $t=0$ situation corresponds to the tiling of weight $r_{1}^{n}$, consisting of all squares of weight $r_{1}$ (not to be confused with one of the $t=1$ tilings with weight $-r_{1}^{n}$ that begins with a square of weight $-r_{1}$ ). Hence for $1 \leq t \leq k$, the total weight of all length $n$-tilings that start with a $t$-omino is $(-1)^{t} e_{t} r_{1}^{n-t}$, so the total weight of all such tilings is the left side of the identity.

To show that the total weight is zero, we find a mate of opposite weight for each tiling as follows. If the leading $t$-omino contains $r_{1}$, then we split that tile into a $(t-1)$-omino followed by a square of weight $r_{1}$. Conversely, if the initial tile does not contain $r_{1}$, then we merge it with the square that follows it, creating a $(t+1)$-omino. For example, the tiling $\left(-r_{2} r_{3} r_{5}\right) r_{1} r_{1} r_{1}$ is paired with the tiling $\left(r_{1} r_{2} r_{3} r_{5}\right) r_{1} r_{1}$ which has opposite weight. See Figure 10. Thus we have a sign reversing involution, resulting in a total weight of zero, as desired.

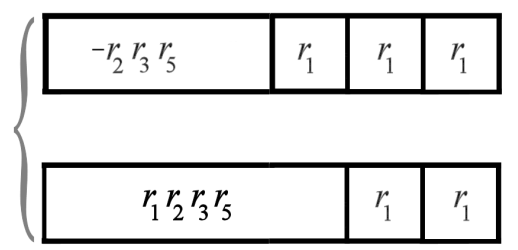

Figure 10: Illustrating the Alternate approach SUGgested By Zeilberger's WORK.

We point out that this involution is very similar to one used by Zeilberger[13] in his proof of Newton's celebrated identities that for $n>0$ and $k>0$,

$$
\sum_{r=0}^{k-1}(-1)^{r}\left(\sum_{1 \leq i_{1}<\cdots<i_{r} \leq n} x_{i_{1}} \cdots x_{i_{r}}\right)\left(\sum_{j=1}^{n} x_{j}^{k-r}\right)+(-1)^{k}\left(\sum_{1 \leq i_{1}<\cdots<i_{k} \leq n} x_{i_{1}} \cdots x_{i_{k}}\right) k=0 .
$$

The situation with multiple roots can be handled in a similar fashion. Here, it suffices to show (by linearity and symmetry) that if $r_{1}$ is a root of multiplicity $m \leq k$, then for $1 \leq j \leq m, h_{n}=\left(\begin{array}{c}n+j-1 \\ j-1\end{array}\right) r_{1}^{n}$ satisfies the previous recurrence. (Note that these $m$ functions span the set of functions of the form $n^{i} r^{n}$ for $i=0,1, \ldots, m-1$.) That is, for fixed $j$ (where $1 \leq j \leq m \leq k$ ) and for $n \geq k$,

$$
\sum_{t=0}^{k}(-1)^{t} e_{t}\left(\begin{array}{c}
n-t+j-1 \\
j-1
\end{array}\right) r_{1}^{n-t}=0,
$$

where $e_{0}=1$ and $e_{t}=\sum_{1 \leq i_{1}<\cdots<i_{t} \leq k} r_{i_{1}} \cdots r_{i_{t}}$.

Since $r_{1}$ has multiplicity $m$, we let $r_{1}=r_{2}=\cdots=r_{m}$. Here, our combinatorial model is a length $n$ tiling that begins with a $t$-omino, $0 \leq t \leq k$, followed by $n-t$ squares, but 
the squares now have weights chosen from $r_{1}, r_{2}, \ldots, r_{j}$ (which we think of for the moment as distinct) in weakly decreasing order. As before, the weight of an initial $t$-omino with label $r_{i_{1}} \cdots r_{i_{t}}$ is $(-1)^{t} r_{i_{1}} \cdots r_{i_{t}}$. Thus $(-1)^{t} e_{t}$ is the total weight of all $t$-ominoes. For fixed $j$, the number of weakly decreasing sequences of length $n-t$ of positive integers that are less than or equal to $j$ is $\left(\begin{array}{c}j+(n-t)-1 \\ n-t\end{array}\right)=\left(\begin{array}{c}n-t+j-1 \\ j-1\end{array}\right)$, so there are $\left(\begin{array}{c}n-t+j-1 \\ j-1\end{array}\right)$ strings of squares (each with weight $r_{1}^{n-t}$ ) that can follow any given $t$-omino. Thus for $0 \leq t \leq k$, the total weight of all length $n$ tilings that start with a $t$-omino is $(-1)^{t} e_{t}\left(\begin{array}{c}n-t+j-1 \\ j-1\end{array}\right) r_{1}^{n-t}$ and the total weight of all such tilings is the left side of the identity.

To prove that the total weight is zero, we use the following sign-reversing involution. Suppose that a tiling begins with a t-omino, followed by a square of weight $r_{h}$, where necessarily $j \geq h$. (If $t=n$, we define $h$ to be zero.) We say that the $t$-omino is splittable if it contains $r_{i}$ where $j \geq i \geq h$. Choosing $i$ as large as possible, we reduce the $t$-omino into a $(t-1)$-omino with root $r_{i}$ removed, followed by a square of weight $r_{i}$. Notice that the new tiling is legal (weakly decreasing square weights), has opposite weight, and the $(t-1)$-omino is not splittable. If the original $t$-omino is not splittable, then all of its labels below $r_{j}$ are less than $r_{h}$ and so we merge the $t$-omino with the square of weight $r_{h}$ to create a $(t+1)$-omino that includes $r_{h}$. Note that this $(t+1)$-omino will be splittable, using the label $r_{h}$. For example, when $n=10, m=8, k=5$, and $j=6$, the tiling $\left(r_{2} r_{3} r_{5} r_{8}\right) r_{3} r_{3} r_{2} r_{2} r_{2} r_{1}$ is splittable, with $h=3$ and $i=5$. It gets paired up with the unsplittable tiling - $\left(r_{2} r_{3} r_{8}\right) r_{5} r_{3} r_{3} r_{2} r_{2} r_{2} r_{1}$ having the same parameters and with opposite weight. See Figure 11. In fact, for any $j, 3 \leq j \leq 8$, the tiling $\left(r_{2} r_{3} r_{5} r_{8}\right) r_{3} r_{3} r_{2} r_{2} r_{2} r_{1}$ is splittable; its mate changes depending on the value of $j$. See Table 1.

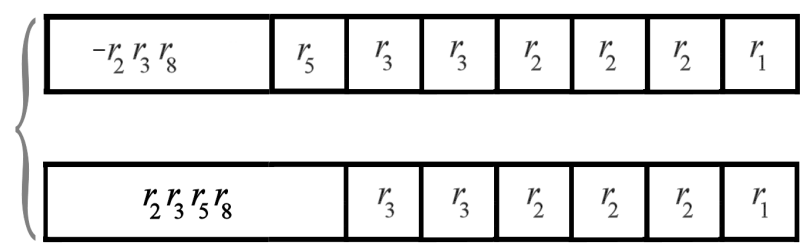

Figure 11: For the parameters $n=10, m=8, k=5$, And $j=6$, the tiling $-\left(r_{2} r_{3} r_{8}\right) r_{5} r_{3} r_{3} r_{2} r_{2} r_{2} r_{1}$ IS UNSPLITTABLE AND GETS MATCHED WITH THE SPLITTABLE TILING $\left(r_{2} r_{3} r_{5} r_{8}\right) r_{3} r_{3} r_{2} r_{2} r_{2} r_{1}$.

The Zeilberger-inspired method gives a concise combinatorial proof. It approaches the problem from the opposite direction - starting with the closed form solution and showing that it satisfies the desired recurrence as opposed to starting with the recurrence and arriving at the closed form solution. The question as to which seems more natural is a matter of taste. We see beauty in both approaches. Acknowledgments Special thanks are due to Ravi Vakil of Stanford University, who provided the initial inspiration for this work, and Michele Intermont, who served as thesis advisor to Halcyon Derks at Kalamazoo College. We are also grateful to Doron Zeilberger, Dan Velleman, and an anonymous referee for suggesting the alternate approach of the last section. 
Table 1: Examples for $n=10, m=8, k=5$, and the tiling $\left(r_{2} r_{3} r_{5} r_{8}\right) r_{3} r_{3} r_{2} r_{2} r_{2} r_{1}$ for varying values of $j \geq 3$.

\begin{tabular}{ccc}
$j$ & Splittable tiling & Matched Unsplittable tiling \\
\hline 3 & $\left(r_{2} r_{3} r_{5} r_{8}\right) r_{3} r_{3} r_{2} r_{2} r_{2} r_{1}$ & $-\left(r_{2} r_{5} r_{8}\right) r_{3} r_{3} r_{3} r_{2} r_{2} r_{2} r_{1}$ \\
4 & $\left(r_{2} r_{3} r_{5} r_{8}\right) r_{3} r_{3} r_{2} r_{2} r_{2} r_{1}$ & $-\left(r_{2} r_{5} r_{8}\right) r_{3} r_{3} r_{3} r_{2} r_{2} r_{2} r_{1}$ \\
5 & $\left(r_{2} r_{3} r_{5} r_{8}\right) r_{3} r_{3} r_{2} r_{2} r_{2} r_{1}$ & $-\left(r_{2} r_{3} r_{8}\right) r_{5} r_{3} r_{3} r_{2} r_{2} r_{2} r_{1}$ \\
6 & $\left(r_{2} r_{3} r_{5} r_{8}\right) r_{3} r_{3} r_{2} r_{2} r_{2} r_{1}$ & $-\left(r_{2} r_{3} r_{8}\right) r_{5} r_{3} r_{3} r_{2} r_{2} r_{2} r_{1}$ \\
7 & $\left(r_{2} r_{3} r_{5} r_{8}\right) r_{3} r_{3} r_{2} r_{2} r_{2} r_{1}$ & $-\left(r_{2} r_{3} r_{8}\right) r_{5} r_{3} r_{3} r_{2} r_{2} r_{2} r_{1}$ \\
8 & $\left(r_{2} r_{3} r_{5} r_{8}\right) r_{3} r_{3} r_{2} r_{2} r_{2} r_{1}$ & $-\left(r_{2} r_{3} r_{5}\right) r_{8} r_{3} r_{3} r_{2} r_{2} r_{2} r_{1}$
\end{tabular}

\section{References}

[1] R. Bellman, Introduction to Matrix Analysis, Society for Industrial and Applied Mathematics, 1987.

[2] A. T. Benjamin and G. P. Dresden, A Combinatorial Proof of Vandermonde's Determinant, Amer. Math. Monthly, 114 (2007), 338-341.

[3] A. T. Benjamin, G. M. Levin, K. Mahlburg, J. J. Quinn, Random approaches to Fibonacci identities, Amer. Math. Monthly, 107.6 (2000), 511-516.

[4] A. T. Benjamin and J. J. Quinn, Alternate Approach to Alternating Sums: A Method to DIE For, College Math. Journal, 39.3 (2008), 191-201.

[5] A. T. Benjamin and J. J. Quinn, Proofs That Really Count: The Art of Combinatorial Proof, Mathematical Association of America, Washington, D.C., 2003.

[6] D. M. Bressoud, Proofs and Confirmations: The Story of the Alternating Sign Matrix Conjecture, Mathematical Association of America, Washington, DC, 1999.

[7] I. Gessel, Tournaments and Vandermonde's determinant, J. of Graph Theory, 3 (1979) 305-307.

[8] D. Poole, Linear Algebra: A Modern Introduction, Brooks/Cole (2003), Chapter 4.

[9] V. Pless, Introduction to the Theory of Error-Correcting Codes, Wiley-Interscience, New York, 1998.

[10] J. J. Quinn, Visualizing Vandermonde's determinant through nonintersecting lattice paths, Journal of Statistical Planning and Inference, 140.8 (2010), 2346-2350.

[11] R. Stanley, Enumerative Combinatorics, Volume 1, Wadsworth \& Brooks/Cole, California, 1986.

[12] D. Zeilberger, A combinatorial approach to matrix algebra, Discrete Math., 56 (1985), 61-72.

[13] D. Zeilberger, A combinatorial proof of Newton's Identities, Discrete Math., 49 (1984), 319 . 